University of Windsor

Scholarship at UWindsor

$12-2014$

\title{
Approaches to improving consistency of interval fuzzy preference relations
}

Wu-Yong Qian

Jiangnan University

Kevin Li

University of Windsor

Zhou-Jing Wang

Zhejiang University of Finance \& Economics

Follow this and additional works at: https://scholar.uwindsor.ca/odettepub

Part of the Business Commons

\section{Recommended Citation}

Qian, Wu-Yong; Li, Kevin; and Wang, Zhou-Jing. (2014). Approaches to improving consistency of interval fuzzy preference relations. Journal of Systems Science and Systems Engineering, 23 (4), 460-479. https://scholar.uwindsor.ca/odettepub/92

This Article is brought to you for free and open access by the Odette School of Business at Scholarship at UWindsor. It has been accepted for inclusion in Odette School of Business Publications by an authorized administrator of Scholarship at UWindsor. For more information, please contact scholarship@uwindsor.ca. 
Approaches to improving consistency of interval fuzzy preference relations Wuyong Qian ${ }^{a}$, Kevin W. Li ${ }^{b}$, and Zhou-Jing Wang ${ }^{c^{*}}$

a School of Business, Jiangnan University, Wuxi 214122, China

${ }^{b}$ Odette School of Business, University of Windsor, Windsor, Ontario N9B 3P4, Canada

c School of Information, Zhejiang University of Finance \& Economics, Hangzhou,

\section{Acknowledgments:}

10 The authors are grateful for the two anonymous referees for their constructive comments

11 that have helped improve the quality and presentation of the article. Zhou-Jing Wang 12 would like to acknowledge the financial support from the National Natural Sciences 13 Foundation of China (NSFC) Grant No: 71271188. Kevin W. Li appreciates the financial 14 support from the Natural Sciences and Engineering Research Council of Canada (NSERC) 15 under its Discovery Grant program and NSFC Grant Nos: 71272129 and 71471056.

\footnotetext{
* Corresponding author, Telephone: +86 571 85043562. Email: wangzj@xmu.edu.cn (Z.J. Wang).
} 
Approaches to improving consistency of interval fuzzy preference relations

3 Abstract

4 This article introduces a consistency index for measuring the consistency level of an 5 interval fuzzy preference relation (IFPR). An approach is then proposed to construct an 6 additive consistent IFPR from a given inconsistent IFPR. By using a weighted averaging 7 method combining the original IFPR and the constructed consistent IFPR, a formula is 8 put forward to repair an inconsistent IFPR to generate an IFPR with acceptable 9 consistency. An iterative algorithm is subsequently developed to rectify an inconsistent 10 IFPR and derive one with acceptable consistency and weak transitivity. The proposed 11 approaches can not only improve consistency of IFPRs but also preserve the initial 12 interval uncertainty information as much as possible. Numerical examples are presented 13 to illustrate how to apply the proposed approaches.

14 Keywords: Interval fuzzy preference relation, Additive consistency, Acceptable 15 consistency, Weak transitivity, Decision making

\section{1. Introduction}

17 In decision analysis, a decision-maker (DM) is often asked to express his/her 18 preference ratings over objects in a pairwise comparison manner (Dong and Saaty 2014).

19 The pairwise comparison among criteria or alternatives in the analytic hierarchy process

20 (AHP) (Saaty 1980) yields multiplicative preference relations, which constitute the basis 21 to derive criteria weights and rank alternatives. To reflect vagueness in human judgment, 22 many researchers have been paying increasing attention to fuzzy preference relations in 23 recent years (Liu X. et al. 2012; Xia et al. 2013).

24 An important research topic in this area is to investigate consistency of preference 25 relations. For fuzzy preference relations, distinct transitivity definitions have been put 26 forward, such as additive transitivity, multiplicative transitivity, weak transitivity, max27 min transitivity, and max-max transitivity ( $\mathrm{Xu}$ 2007). Let $R=\left(r_{i j}\right)_{n \times n}$ be a fuzzy 28 preference relation, if $r_{i j}-r_{j i}$ is interpreted as the intensity of the DM's preference of the 29 object $x_{i}$ over $x_{j}$, then additive consistency is a sensible vehicle to verify whether the 
30 DM's judgments are contradiction-free; if the DM denotes $r_{i j} / r_{j i}$ as its preference

31 intensity for $x_{i}$ vs. $x_{j}$, then multiplicative consistency is an appropriate tool. The focus

32 of this paper is concerned with additive transitivity, which is regarded as a parallel

33 concept to the multiplicative consistent property in AHP (Herrera-Viedma et al. 2004;

34 Liu X. 2012; Xu et al. 2014) and is widely employed to characterize consistency of fuzzy

35 preference relations (Chen and Chao 2012; Chiclana et al., 2007; Herrera-Viedma et al.

36 2004, 2007; Liu X. et al. 2012; Ma et al. 2006; Xu et al. 2014). Based on additive

37 transitivity properties, some authors have proposed different approaches to improve

38 consistency of inconsistent fuzzy preference relations furnished by the DM. For example,

39 Herrera-Viedma et al. (2004) put forward an approach to construct a fuzzy preference

40 relation with additive consistency from a set of $n-1$ preference values. Ma et al. (2006)

41 present two methods to examine weak transitivity of a fuzzy preference relation with

42 strict pairwise comparison judgments, and develop an algorithm to repair an inconsistent

43 fuzzy preference relation to reach weak transitivity. Herrera-Viedma et al. (2007)

44 introduce a consistency index to measure the consistency level $(C L)$ of a fuzzy preference

45 relation and furnish a concept of fully additive consistency when $C L=1$. Liu X. et al.

46 (2012) consider incomplete fuzzy preference relations and develop a least square model

47 to complete an incomplete fuzzy preference relation and rectify its inconsistency based

48 on additive transitivity.

49 On the other hand, due to complexity and uncertainty in many decision problems, it is

50 hard for a DM to express his/her preference over objects with crisp values (Durbach and

51 Stewart 2012; Li and Chen 2014; Yu and $\mathrm{Xu}$ 2014). In this case, it is often more natural

52 to use interval fuzzy preference relations (IFPRs). The concept of IFPRs is introduced by

$53 \mathrm{Xu}$ (2004), in which judgment data are given as interval fuzzy numbers to characterize a

54 DM's preference degree or intensity of one object over another. In order to obtain 55 reasonable priority weights, consistency and acceptable consistency of IFPRs have been

56 studied and different methods have been designed for generating priority weights based

57 on IFPRs. For instance, $\mathrm{Xu}$ and Chen (2008) define additive and multiplicative consistent

58 IFPRs, in which the consistency conditions are established without accounting for

59 transitivity among three or more judgment data. Based on $\mathrm{Xu}$ and Chen's additive and

60 multiplicative consistency, some authors have devised different methods for generating 
61 priority weights from IFPRs such as Genç et al. (2010), Lan et al. (2012), Xia and Xu 62 (2014), to name a few. Xu (2011) further proposes an approach to construct additive or 63 multiplicative consistent IFPRs by minimizing deviation between the initial and 64 constructed IFPRs. Xu et al. (2014) and Hu et al. (2014) propose revised definitions for 65 the additive consistency given by $\mathrm{Xu}$ and Chen (2008). Liu F. et al. (2012) adopt two converted fuzzy preference relations to define an additive consistent IFPR, and develop

67 an algorithm for deriving priority weights from IFPRs. Wang and Li (2012) introduce 68 new additive and multiplicative consistency definitions for IFPRs based on interval 69 arithmetic. Wang and $\mathrm{Li}$ (2014) develop a multi-step goal programming method for group 70 decision making with incomplete IFPRs.

71 Consistency of preference relations plays an important role in reaching a reasonable 72 decision result. Nevertheless, it is often a challenge for a DM to provide a consistent 73 IFPR in many real-world decision situations. It is natural that highly inconsistent 74 judgment matrices may lead to misleading decision result. For IFPRs with low 75 consistency levels, they should be returned to the DMs for an update. If the DMs are 76 unavailable or unwilling to revise their original judgment information, it is helpful to 77 have an automated process to improve consistency of the original IFPRs furnished by the 78 DMs. In the case that the DMs are available to update their decision input, the results of 79 improved IFPRs can also serve as a valuable feedback and benchmark for the DMs in 80 updating their judgment. Although the approach in $\mathrm{Xu}$ (2011) is able to construct a 81 consistent IFPR, the consistency definitions are based on crisp weights and the derived 82 consistent IFPR may result in significant loss of information (for instance, the uncertainty 83 reflected in the interval width of the judgment may be substantially changed in the 84 conversion process). In addition, for the consistency definitions given by $\mathrm{Xia}$ and $\mathrm{Xu}$ 85 (2011) and Liu F. et al. (2012), Wang and Chen (2014) point out their technical 86 deficiency as the consistency status of an IFPR therein is sensitive to alternative 87 permutations. The fundamental motivation of this research is to address the aforesaid 88 issues. By adopting the additive consistency notion proposed by Wang and Li (2012), this 89 study focuses on improving consistency of IFPRs. The contributions of this article are 90 threefold: we first define a consistency index for IFPRs, then put forward a formula to 91 construct an additive consistent IFPR based on an inconsistent input, finally, we develop 
92 a method and an algorithm to rectify an inconsistent IFPR. More specifically, a 93 consistency index is first defined to measure the consistency level of an IFPR. For an 94 inconsistent IFPR, an approach is then proposed to construct an additive consistent IFPR, 95 which is employed as a reference to improve consistency of the given IFPR. By using a 96 weighted averaging scheme combining the original IFPR and the constructed consistent 97 IFPR, a method is put forward to repair an inconsistent IFPR to yield an IFPR with 98 acceptable consistency. A further algorithm is developed to rectify an inconsistent IFPR 99 to generate an IFPR with both acceptable consistency and weak transitivity.

100 The remainder of this paper is organized as follows. Section 2 provides preliminaries 101 on consistent IFPRs and comparison of interval numbers. Section 3 defines a consistency 102 index for IFPRs. In Section 4, an approach is proposed to construct an additive consistent 103 IFPR based on any given IFPR. Section 5 presents two approaches to improving 104 consistency of IFPRs. Finally, concluding remarks are furnished in Section 6.

\section{2. Preliminaries}

106 This section presents basic concepts of additive consistency and weak transitivity of IFPRs as well as comparison of interval numbers.

110 Let $I$ be a real closed interval, $D(I)=\left\{\left[a^{-}, a^{+}\right]: a^{-} \leq a^{+}, a^{-}, a^{+} \in I\right\}$. For any $x \in I$, define $111 x=[x, x]$.

$112 \mathrm{Xu}$ (2004) defines IFPRs where judgment data are expressed as interval fuzzy 113 numbers to characterize a DM's preference degree of one object over another.

114 Definition 2.1 (Xu 2004) An interval fuzzy preference relation (IFPR) $\bar{R}$ on the set $115 X$ is characterized by an interval fuzzy preference matrix $\bar{R}=\left(\bar{r}_{i j}\right)_{n \times n} \subset X \times X$, where

$$
\bar{r}_{i j}=\left[r_{i j}^{-}, r_{i j}^{+}\right] \in D([0,1]), \bar{r}_{j i}=1-\bar{r}_{i j}=\left[1-r_{i j}^{+}, 1-r_{i j}^{-}\right], \bar{r}_{i i}=[0.5,0.5], i, j=1,2, \ldots, n
$$

117 and $\bar{r}_{i j}$ indicates the interval-valued fuzzy preference of $x_{i}$ over $x_{j} \cdot r_{i j}^{-}$and $r_{i j}^{+}$are the 118 lower and upper bounds of $\bar{r}_{i j}$, respectively.

119 As commented in Section 1, the additive consistency definitions introduced by $\mathrm{Xu}$ and 120 Chen (2008), Xu et al. (2014) and Hu et al. (2014) are based on crisp weights, and the 
121 consistency condition established therein fails to account for transitivity among three or 122 more judgment data. To address this issue, Wang and Li (2012) put forward a new 123 additive consistency notion for IFPRs by using interval arithmetic and the definition is 124 furnished below.

125 Definition 2.2 (Wang and Li 2012) An IFPR $\bar{R}=\left(\bar{r}_{i j}\right)_{n \times n}$ is additive consistent if the 126 following additive transitivity is satisfied

$$
\bar{r}_{i j}+\bar{r}_{j k}+\bar{r}_{k i}=\bar{r}_{k j}+\bar{r}_{j i}+\bar{r}_{i k} \quad \text { for all } i, j, k=1,2, \ldots, n
$$

128 To compare two interval numbers $\bar{a}=\left[a^{-}, a^{+}\right]$and $\bar{b}=\left[b^{-}, b^{+}\right]$, where $a^{-}, b^{-} \geq 0$, the 129 notion of likelihood is introduced. Let $\bar{a} \geq \bar{b}$ represent that $\bar{a}$ is no smaller than $\bar{b}$. The 130 likelihood of $\bar{a} \geq \bar{b}$ is defined as (Xu and Chen 2008)

$$
p(\bar{a} \geq \bar{b})=\frac{\max \left\{0, a^{+}-b^{-}\right\}-\max \left\{0, a^{-}-b^{+}\right\}}{a^{+}-a^{-}+b^{+}-b^{-}}
$$

Some useful properties about likelihood $p(\bar{a} \geq \bar{b})$ are summarized as follows:

(a) $0 \leq p(\bar{a} \geq \bar{b}) \leq 1$;

$$
\text { (b) } p(\bar{a} \geq \bar{b})+p(\bar{b} \geq \bar{a})=1 \text {; }
$$

(c) $p(\bar{a} \geq \bar{b})=1$ if and only if $a^{-} \geq b^{+}$;

(d) $p(\bar{a} \geq \bar{b})=0$ if and only if $a^{+} \leq b^{-}$;

138 only if $\frac{a^{-}+a^{+}}{2}=\frac{b^{-}+b^{+}}{2}$;

139 (f) For any interval numbers $\bar{a}, \bar{b}$ and $\bar{c}$, if $p(\bar{a} \geq \bar{b}) \geq 0.5$ and $p(\bar{b} \geq \bar{c}) \geq 0.5$, then $140 \quad p(\bar{a} \geq \bar{c}) \geq 0.5$.

141 According to the aforesaid properties of the likelihood concept, for an IFPR $142 \bar{R}=\left(\bar{r}_{i j}\right)_{n \times n}, p\left(\bar{r}_{i j} \geq[0.5,0.5]\right)=0.5$ indicates a DM's indifference between $x_{i}$ and $x_{j}$, $143 p\left(\bar{r}_{i j} \geq[0.5,0.5]\right)>0.5$ signifies that $x_{i}$ is preferred to $x_{j}$ with a degree of $p\left(\bar{r}_{i j} \geq[0.5,0.5]\right)$, $144 p\left(\bar{r}_{i j} \geq[0.5,0.5]\right)<0.5$ describes that $x_{j}$ is preferred to $x_{i}$ with a degree of $1451-p\left(\bar{r}_{i j} \geq[0.5,0.5]\right), p\left(\bar{r}_{i j} \geq[0.5,0.5]\right)=1$ means that $x_{i}$ is absolutely preferred to $x_{j}$, and 

$p\left(\bar{r}_{i j} \geq[0.5,0.5]\right)=0$ expresses that $x_{j}$ is absolutely preferred to $x_{i}$.

147 Based on the likelihood definition and properties, Wang and Li (2012) introduce weak transitivity for IFPRs as follows.

149 Definition 2.3 (Wang and Li 2012) An IFPR $\bar{R}=\left(\bar{r}_{i j}\right)_{n \times n}$ is weakly transitive if $p\left(\bar{r}_{i k} \geq[0.5,0.5]\right) \geq 0.5$ and $p\left(\bar{r}_{k j} \geq[0.5,0.5]\right) \geq 0.5$ imply $p\left(\bar{r}_{i j} \geq[0.5,0.5]\right) \geq 0.5$, for all $i, j, k=1,2, \ldots, n$.

Base on Definition 2.2, Wang (2014) provides the following property to judge whether an IFPR is consistent.

Lemma 2.1 (Wang 2014) An IFPR $\bar{R}=\left(\bar{r}_{i j}\right)_{n \times n}$ is additive consistent if and only if

$$
r_{i j}^{-}+r_{i j}^{+}+r_{j k}^{-}+r_{j k}^{+}+r_{k i}^{-}+r_{k i}^{+}=3 \quad \forall i, j, k=1,2, \ldots, n
$$

\section{Consistency measure}

By Lemma 2.1, if $\bar{R}=\left(\bar{r}_{i j}\right)_{n \times n}$ is an additive consistent IFPR, we have $r_{i j}^{-}+r_{i j}^{+}+r_{j k}^{-}+r_{j k}^{+}+r_{k i}^{-}+r_{k i}^{+}=3 \forall i, j, k=1,2, \ldots, n$. However, if $\bar{R}$ is inconsistent, the preference values in $\bar{R}$ will not satisfy (3.1). In other words, there exist some differences between $r_{i j}^{-}+r_{i j}^{+}+r_{j k}^{-}+r_{j k}^{+}+r_{k i}^{-}+r_{k i}^{+}$and 3 for some $i, j, k=1,2, \ldots, n$. As $0 \leq r_{i j}^{-} \leq r_{i j}^{+} \leq 1$ for all $i, j=1,2, \ldots, n$, one has $0 \leq\left|r_{i j}^{-}+r_{i j}^{+}+r_{j k}^{-}+r_{j k}^{+}+r_{k i}^{-}+r_{k i}^{+}-3\right| \leq 3$. Therefore, we can define a consistency measure for an IFPR as follows. Definition 3.1 A consistency index of an IFPR $\bar{R}=\left(\bar{r}_{i j}\right)_{n \times n}$ is defined as

$$
C I(\bar{R})=1-\frac{1}{3 n(n-1)(n-2)} \sum_{i=1}^{n} \sum_{j=1, j \neq i}^{n} \sum_{k=1, k \neq i, j}^{n}\left|r_{i j}^{-}+r_{i j}^{+}+r_{j k}^{-}+r_{j k}^{+}+r_{k i}^{-}+r_{k i}^{+}-3\right|
$$
It is obvious that $0 \leq C I(\bar{R}) \leq 1$. If $C I(\bar{R})=1$, then the IFPR $\bar{R}$ is additive consistent;

167 IFPR. According to the actual situation, if a DM can accept limited inconsistency in the 168 judgment, he/she may give a consistency threshold $0<t<1$ for $C I(\bar{R})$. This threshold 169 presumably reflects the DM's tolerance for inconsistency and should be furnished by the DM upon examining the specific decision circumstances. If $\operatorname{CI}(\bar{R}) \geq t$, the $\operatorname{IFPR} \bar{R}$ is 
171 deemed acceptably consistent; otherwise, the consistency level of $\bar{R}$ is considered

172 unacceptable and, hence, $\bar{R}$ should be rectified to ensure rationality of decisions.

173 If $r_{i j}^{-}=r_{i j}^{+}, i, j=1,2, \ldots, n$, the IFPR $\bar{R}$ is reduced to a fuzzy preference relation, and

174 (3.1) is equivalent to the consistency index of a complete fuzzy preference relation 175 proposed by Herrera-Viedma et al. (2007).

176 As per the additive consistency of IFPRs in Definition 2.2 and weak transitivity of 177 IFPRs in Definition 2.3, we have the following theorem.

178 Theorem 3.1 If an IFPR $\bar{R}=\left(\bar{r}_{i j}\right)_{n \times n}$ is additive consistent, then $\bar{R}$ is weakly transitive.

179 Proof. According to property (e) of the likelihood concept in Section 2, if $180 p\left(\bar{r}_{i k} \geq[0.5,0.5]\right) \geq 0.5$ and $p\left(\bar{r}_{k j} \geq[0.5,0.5]\right) \geq 0.5$, we have $r_{i k}^{-}+r_{i k}^{+} \geq(0.5+0.5)=1$ and $181 r_{k j}^{-}+r_{k j}^{+} \geq(0.5+0.5)=1, \forall i, j, k \in\{1,2, \ldots, n\}$. As per the reciprocal property of $\bar{r}_{j i}=1-\bar{r}_{i j}$, 182 one can get $1-r_{k i}^{+}+1-r_{k i}^{-} \geq 1$ and $1-r_{j k}^{+}+1-r_{j k}^{-} \geq 1, \forall i, j, k \in\{1,2, \ldots, n\}$. It follows that $183 \quad r_{k i}^{-}+r_{k i}^{+} \leq 1$ and $r_{j k}^{-}+r_{j k}^{+} \leq 1, \forall i, j, k \in\{1,2, \ldots, n\}$.

184 On the other hand, as $\bar{R}$ is additive consistent, it follows from Lemma 2.1 $185 r_{i j}^{-}+r_{i j}^{+}+r_{j k}^{-}+r_{j k}^{+}+r_{k i}^{-}+r_{k i}^{+}=3 \forall i, j, k=1,2, \ldots, n$. Then, one has $r_{i j}^{-}+r_{i j}^{+} \geq 1$, i.e., $186 \frac{r_{i j}^{-}+r_{i j}^{+}}{2} \geq 0.5$. As per the property (e) of the likelihood concept, we get $187 p\left(\bar{r}_{i j} \geq[0.5,0.5]\right) \geq 0.5$. Therefore, $\bar{R}=\left(\bar{r}_{i j}\right)_{n \times n}$ is weakly transitive.

188 For any two IFPRs $\bar{R}=\left(\bar{r}_{i j}\right)_{n \times n}=\left(\left[r_{i j}^{-}, r_{i j}^{+}\right]\right)_{n \times n}$ and $\bar{R}^{\prime}=\left(\bar{r}_{i j}^{\prime}\right)_{n \times n}=\left(\left[r_{i j}^{\prime-}, r_{i j}^{\prime+}\right]\right)_{n \times n}$, let

$$
d\left(\bar{R}, \bar{R}^{\prime}\right)=\frac{1}{2 n(n-1)} \sum_{i=1}^{n} \sum_{j=1, j \neq i}^{n}\left(\left|r_{i j}^{-}-r_{i j}^{\prime-}\right|+\left|r_{i j}^{+}-r_{i j}^{\prime+}\right|\right)
$$

190 denote the mean absolute deviation for all off-diagonal intervals between $\bar{R}$ and $\bar{R}^{\prime}$. The 191 smaller the value $d\left(\bar{R}, \bar{R}^{\prime}\right)$, the closer the $\bar{R}$ is to $\bar{R}^{\prime}$. Especially, if $d\left(\bar{R}, \bar{R}^{\prime}\right)=0, \bar{R}$ is 192 the same as $\bar{R}^{\prime}$.

\section{4. An approach to constructing consistent IFPRs}

194 This section develops a framework to construct an additive consistent IFPR based on 195 any given inconsistent IFPR. 
196 For a given IFPR $\bar{R}=\left(\bar{r}_{i j}\right)_{n \times n}$, define the $(i, j)$ entry of $\hat{\bar{R}}=\left(\hat{\bar{r}}_{i j}\right)_{n \times n}=\left(\left[\hat{r}_{i j}^{-}, \hat{r}_{i j}^{+}\right]\right)_{n \times n}$ as 197 follows

198

$$
\begin{aligned}
& \hat{\bar{r}}_{i j}=\left[\hat{r}_{i j}^{-}, \hat{r}_{i j}^{+}\right]=\left[0.5+\frac{1}{2 n}\left(\sum_{l=1}^{n} r_{i l}^{-}+\sum_{l=1}^{n} r_{i l}^{+}-\sum_{l=1}^{n} r_{j l}^{-}-\sum_{l=1}^{n} r_{j l}^{+}\right)-\frac{r_{i j}^{+}-r_{i j}^{-}}{2},\right. \\
& \left.0.5+\frac{1}{2 n}\left(\sum_{l=1}^{n} r_{i l}^{-}+\sum_{l=1}^{n} r_{i l}^{+}-\sum_{l=1}^{n} r_{j l}^{-}-\sum_{l=1}^{n} r_{j l}^{+}\right)+\frac{r_{i j}^{+}-r_{i j}^{-}}{2}\right]
\end{aligned}
$$

199 for all $i, j=1,2, \ldots, n$. The following two theorems reveal some useful properties of $\hat{\hat{r}_{i j}}$. Theorem 4.1 Let $\bar{R}=\left(\bar{r}_{i j}\right)_{n \times n}$ be an IFPR and $\overline{\bar{r}}_{i j}=\left[\hat{r}_{i j}^{-}, \hat{r}_{i j}^{+}\right](i, j=1,2, \ldots, n)$ be 201 defined by (4.1), then

(i) $\hat{r}_{i j}^{-} \leq \hat{r}_{i j}^{+}, \hat{r}_{i i}^{-}=\hat{r}_{i i}^{+}=0.5 \forall i, j=1,2, \ldots, n$.

(iii) $\hat{r}_{i j}^{+}-\hat{r}_{i j}^{-}=r_{i j}^{+}-r_{i j}^{-} \quad \forall i, j=1,2, \ldots, n$.

$$
\text { (iv) } \hat{\bar{r}}_{i j}+\hat{\bar{r}}_{j k}+\hat{\bar{r}}_{k i}=\hat{\bar{r}}_{k j}+\hat{\bar{r}}_{j i}+\hat{\bar{r}}_{i k} \forall i, j, k=1,2, \ldots, n \text {. }
$$

Proof. (i) - (iii) can be immediately derived from (4.1) and, hence, the proof is only provided for (iv).

209 follows from (4.1) that

$$
\begin{aligned}
& \hat{r}_{i j}^{-}+\hat{r}_{j k}^{-}+\hat{r}_{k i}^{-}=\frac{3}{2}-\frac{r_{i j}^{+}-r_{i j}^{-}+r_{j k}^{+}-r_{j k}^{-}+r_{k i}^{+}-r_{k i}^{-}}{2}=\frac{3}{2}-\frac{1-r_{j i}^{-}-\left(1-r_{j i}^{+}\right)+1-r_{k j}^{-}-\left(1-r_{k j}^{+}\right)+1-r_{i k}^{-}-\left(1-r_{i k}^{+}\right)}{2} \\
& =\frac{3}{2}-\frac{r_{k j}^{+}-r_{k j}^{-}+r_{j i}^{+}-r_{j i}^{-}+r_{i k}^{+}-r_{i k}^{-}}{2}=\hat{r}_{k j}^{-}+\hat{r}_{j i}^{-}+\hat{r}_{i k}^{-}
\end{aligned}
$$

211 Similarly, from (4.1), one can obtain $\hat{r}_{i j}^{+}+\hat{r}_{j k}^{+}+\hat{r}_{k i}^{+}=\hat{r}_{k j}^{+}+\hat{r}_{j i}^{+}+\hat{r}_{i k}^{+}$. Therefore,

$212 \hat{\overline{r_{i j}}}+\hat{\bar{r}}_{j k}+\hat{\bar{r}}_{k i}=\hat{\bar{r}}_{k j}+\hat{\bar{r}}_{j i}+\hat{\bar{r}}_{i k}$. This completes the proof of Theorem 4.1.

$$
\text { Theorem } 4.2 \text { If } \bar{R}=\left(\bar{r}_{i j}\right)_{n \times n} \text { is an additive consistent IFPR, then } \hat{\bar{r}}_{i j}=\bar{r}_{i j}
$$
$\forall i, j=1,2, \ldots, n$.

215 Proof. Since $\bar{R}$ is additive consistent, it follows from Lemma 2.1 that

$$
r_{i l}^{-}+r_{i l}^{+}+r_{l j}^{-}+r_{l j}^{+}=3-\left(r_{j i}^{-}+r_{j i}^{+}\right) \forall i, j, l=1,2, \ldots, n
$$


Then,

$$
\begin{aligned}
& \sum_{l=1}^{n} r_{i l}^{-}+\sum_{l=1}^{n} r_{i l}^{+}-\sum_{l=1}^{n} r_{j l}^{-}-\sum_{l=1}^{n} r_{j l}^{+}=\sum_{l=1}^{n}\left(r_{i l}^{-}+r_{i l}^{+}-r_{j l}^{-}-r_{j l}^{+}\right)=\sum_{l=1}^{n}\left(r_{i l}^{-}+r_{i l}^{+}-\left(1-r_{l j}^{+}\right)-\left(1-r_{l j}^{-}\right)\right) \\
& =\sum_{l=1}^{n}\left(r_{i l}^{-}+r_{i l}^{+}+r_{l j}^{-}+r_{l j}^{+}-2\right)=\sum_{l=1}^{n}\left(3-\left(r_{j i}^{-}+r_{j i}^{+}\right)-2\right)=\sum_{l=1}^{n}\left(1-\left(1-r_{i j}^{+}+1-r_{i j}^{-}\right)\right) \\
& =\sum_{l=1}^{n}\left(r_{i j}^{-}+r_{i j}^{+}-1\right)=n\left(r_{i j}^{-}+r_{i j}^{+}\right)-n
\end{aligned}
$$

As per (4.1), we have $\hat{r}_{i j}^{-}=0.5+\frac{\left(r_{i j}^{-}+r_{i j}^{+}\right)}{2}-0.5-\frac{\left(r_{i j}^{+}-r_{i j}^{-}\right)}{2}=r_{i j}^{-}$and $\hat{r}_{i j}^{+}=0.5+\frac{\left(r_{i j}^{-}+r_{i j}^{+}\right)}{2}-$

$220 \quad 0.5+\frac{\left(r_{i j}^{+}-r_{i j}^{-}\right)}{2}=r_{i j}^{+}$. It is verified that $\hat{\bar{r}_{i j}}=\overline{r_{i j}}$.

221 Theorem 4.1 demonstrates that $\hat{\bar{R}}$ is an additive consistent IFPR if $0 \leq \hat{r}_{i j}^{-} \leq \hat{r}_{i j}^{+} \leq 1$

$222 \forall i, j=1,2, \ldots, n$ and the interval width of each element in $\hat{\bar{R}}$ remains the same for the

223 corresponding element in $\bar{R}$. Theorem 4.2 further confirms that $\hat{\bar{R}}=\bar{R}$ if $\bar{R}$ is additive

224 consistent. Therefore, a simple way to tell whether $\bar{R}$ is additive consistent is to compute

$225 \hat{\bar{r}_{i j}} \forall i<j=1,2, \ldots, n$ and examine if $\hat{\bar{r}}_{i j}=\bar{r}_{i j}$. This judgment method only needs to compute

$226 n \cdot(n-1) / 2$ values in contrast to the direct application of Definition 2.2 that has to

227 entertain $n^{3}$ data.

228 If $\bar{R}$ is not additive consistent, we may obtain a matrix $\hat{\bar{R}}=\left(\left[\hat{r}_{i j}^{-}, \hat{r}_{i j}^{+}\right]\right)_{n \times n}$ with entries

229 outside $D([0,1])$. In this case, $\hat{\bar{R}}$ is not an IFPR. To construct an additive consistent

230 IFPR from $\bar{R}$, interval values $\left[\hat{r}_{i j}^{-}, \hat{r}_{i j}^{+}\right]$have to be further converted to intervals on

$231 D([0,1])$. This conversion process should presumably preserve additive transitivity and

232 the complementary property in the sense of $\hat{\bar{r}}_{j i}=1-\hat{\bar{r}}_{i j}$.

$233 \quad$ Let

234

$$
c= \begin{cases}1, & \text { if } \hat{r}_{i j}^{+} \leq 1, \forall i, j=1,2, \ldots, n \\ \max \left\{\hat{r}_{i j}^{+} \mid \hat{r}_{i j}^{+}>1, i, j=1,2, \ldots, n\right\}, & \text { Otherwise }\end{cases}
$$

235 It is obvious that $c \geq 1$, and $\hat{r}_{i j}^{-} \leq \hat{r}_{i j}^{+} \leq c \forall i, j=1,2, \ldots, n$. According to Theorem 4.1, we 236 have $\hat{r}_{i j}^{-}=1-\hat{r}_{j i}^{+} \quad \forall i, j=1,2, \ldots, n$. Thus, one can obtain $1-c \leq 1-\hat{r}_{j i}^{+}=\hat{r}_{i j}^{-} \leq \hat{r}_{i j}^{+} \leq c$ 
$237 \forall i, j=1,2, \ldots, n$. Therefore, all elements in $\hat{\bar{R}}=\left(\left[\hat{r}_{i j}^{-}, \hat{r}_{i j}^{+}\right]\right)_{n \times n}$ should lie between $238[1-c, 1-c]$ and $[c, c]$, i.e., $\left[\hat{r}_{i j}^{-}, \hat{r}_{i j}^{+}\right] \in D([1-c, c]) \forall i, j=1,2, \ldots, n$.

239 In order to convert $\hat{\bar{R}}$ into an additive consistent IFPR, an appropriate transformation 240 function $\varphi: D([1-c, c]) \rightarrow D([0,1])$ should possess the following properties:

(i) $\varphi([1-c, 1-c])=[0,0]$.

$$
\text { (ii) } \varphi([c, c])=[1,1] \text {. }
$$

(iii) $\varphi([0.5,0.5])=[0.5,0.5]$

(iv) $\varphi(\bar{x})=1-\varphi(1-\bar{x}) \quad \forall \bar{x} \in D([1-c, c])$.

(v) $\forall \bar{x}_{1}, \bar{x}_{2}, \bar{x}_{3}, \bar{y}_{1}, \bar{y}_{2}, \bar{y}_{3} \in D([1-c, c])$, if $\bar{x}_{1}+\bar{x}_{2}+\bar{x}_{3}=\bar{y}_{1}+\bar{y}_{2}+\bar{y}_{3}$, then $\varphi\left(\bar{x}_{1}\right)+\varphi\left(\bar{x}_{2}\right)+$ $\varphi\left(\bar{x}_{3}\right)=\varphi\left(\bar{y}_{1}\right)+\varphi\left(\bar{y}_{2}\right)+\varphi\left(\bar{y}_{3}\right)$.

(i) and (ii) ensure that the transformation function should be able to convert the smallest interval $[1-c, 1-c]$ and the largest interval $[c, c]$ into $[0,0]$ and $[1,1]$ on $D([0,1])$, respectively. (iii) expects that $\varphi($.$) maintain indifference to be [0.5,0.5]$ after conversion. (iv) requires that $\varphi($.) keep the complementary property in the sense of interval arithmetic. The last desired property (v) guarantees that additive transitivity remains after $\varphi($.$) is applied. If a transformation function satisfies these five properties,$ the following theorem immediately follows.

Theorem 4.3 Let $\bar{R}=\left(\bar{r}_{i j}\right)_{n \times n}$ be an IFPR, then $\hat{\bar{R}}^{\prime}=\left(\varphi\left(\left[\hat{r}_{i j}^{-}, \hat{r}_{i j}^{+}\right]\right)\right)_{n \times n}$ is an additive 255 consistent IFPR.

256 Next, similar to the function furnished in Herrera-Viedma et al. (2004) for fuzzy 257 preference relations, the following transformation function with the aforesaid desired 258 properties is provided for handling IFPRs. Let

$$
\varphi(\bar{x})=\left[\frac{x^{-}+c-1}{2 c-1}, \frac{x^{+}+c-1}{2 c-1}\right] \quad \forall \bar{x}=\left[x^{-}, x^{+}\right] \in D([1-c, c])
$$

260 It is apparent that this function satisfies (i), (ii) and (iii). As for (iv), since 261

$$
\begin{aligned}
\varphi(\bar{x}) & =\left[\frac{x^{-}+c-1}{2 c-1}, \frac{x^{+}+c-1}{2 c-1}\right]=1-\left[\frac{1-x^{+}+c-1}{2 c-1}, \frac{1-x^{-}+c-1}{2 c-1}\right]=1-\varphi\left(\left[1-x^{+}, 1-x^{-}\right]\right) \\
& =1-\varphi(1-\bar{x})
\end{aligned}
$$


262 (iv) is thus verified. Moreover, if $\bar{x}_{1}+\bar{x}_{2}+\bar{x}_{3}=\bar{y}_{1}+\bar{y}_{2}+\bar{y}_{3},(\mathrm{v})$ is confirmed as

$$
\begin{aligned}
& \varphi\left(\bar{x}_{1}\right)+\varphi\left(\bar{x}_{2}\right)+\varphi\left(\bar{x}_{3}\right)=\left[\frac{x_{1}^{-}+x_{2}^{-}+x_{3}^{-}+3 c-3}{2 c-1}, \frac{x_{1}^{+}+x_{2}^{+}+x_{3}^{+}+3 c-3}{2 c-1}\right] \\
& =\left[\frac{y_{1}^{-}+y_{2}^{-}+y_{3}^{-}+3 c-3}{2 c-1}, \frac{y_{1}^{+}+y_{2}^{+}+y_{3}^{+}+3 c-3}{2 c-1}\right]=\varphi\left(\bar{y}_{1}\right)+\varphi\left(\bar{y}_{2}\right)+\varphi\left(\bar{y}_{3}\right)
\end{aligned}
$$

264 After applying the transformation function (4.3), $\hat{\bar{r}}_{i j}$ is converted to $\hat{\bar{r}}_{i j}^{\prime}$ as shown below

$$
\hat{\bar{r}}_{i j}^{\prime}=\left[\hat{r}_{i j}^{-}, \hat{r}_{i j}^{\prime+}\right]=\varphi\left(\left[\hat{r}_{i j}^{-}, \hat{r}_{i j}^{+}\right]\right)=\left[\frac{\hat{r}_{i j}^{-}+c-1}{2 c-1}, \frac{\hat{r}_{i j}^{+}+c-1}{2 c-1}\right]
$$

266 where $c$ is defined by (4.2).

267 Corollary 4.1 Assume that the elements of $\hat{\bar{R}}^{\prime}=\varphi(\hat{\bar{R}})=\left(\hat{\bar{r}}_{i j}^{\prime}\right)_{n \times n}$ are defined by (4.4),

268 then $\hat{\bar{R}}^{\prime}$ is an additive consistent IFPR and $\hat{r}_{i j}^{++}-\hat{r}_{i j}^{-}=\frac{r_{i j}^{+}-r_{i j}^{-}}{2 c-1} \forall i, j=1,2, \ldots, n$.

269 Proof. It can be obtained from Theorem 4.3 that $\hat{\bar{R}}^{\prime}$ is an additive consistent IFPR. By 270 Theorem 4.1, we have $\hat{r}_{i j}^{+}-\hat{r}_{i j}^{-}=r_{i j}^{+}-r_{i j}^{-} \quad \forall i, j=1,2, \ldots, n$. It follows that $\hat{r}_{i j}^{++}-\hat{r}_{i j}^{-}=$ $271 \quad \frac{\hat{r}_{i j}^{+}-\hat{r}_{i j}^{-}}{2 c-1}=\frac{r_{i j}^{+}-r_{i j}^{-}}{2 c-1}$.

272 Corollary 4.1 shows that an additive consistent IFPR can be constructed from any 273 given $\bar{R}$. If $\bar{R}$ is additive consistent, the constructed IFPR $\hat{\bar{R}}^{\prime}=\bar{R}$. For a given additive 274 inconsistent IFPR $\bar{R}$, if $c=1$, the interval widths of each element in the constructed 275 consistent IFPR $\hat{\bar{R}}^{\prime}$ is equal to that of the corresponding element in the original IFPR $\bar{R}$; 276 if $c>1$, the proposed method scales down the interval widths of each element in $\bar{R}$ by a 277 common factor $\frac{1}{2 c-1}$. As the width of an interval is a natural way to gauge interval 278 uncertainty, the constructed consistent IFPR $\hat{\bar{R}}^{\prime}$ is able to keep the original interval 279 uncertainty in terms of their widths if all converted elements in $\hat{\bar{R}}$ fall within $D([0,1])$. In 280 the case that some elements in $\hat{\bar{R}}$ have an upper bound above 1 or a lower bound below 0 , 281 this conversion process yields an $\hat{\bar{R}}^{\prime}$ that proportionally scales down the largest upper 
282 bound to 1 and scales up the smallest negative lower bound to 0 , thereby preserving 283 interval uncertainty as much as possible.

284 Next, a numerical example is presented to show how to apply the proposed method.

285 Example 1. Consider the following three IFPRs,

286

$$
\bar{R}_{1}=\left[\begin{array}{llll}
{[0.5,0.5]} & {[0.4,0.5]} & {[0.5,0.6]} & {[0.4,0.5]} \\
{[0.5,0.6]} & {[0.5,0.5]} & {[0.5,0.6]} & {[0.6,0.7]} \\
{[0.4,0.5]} & {[0.4,0.5]} & {[0.5,0.5]} & {[0.6,0.8]} \\
{[0.5,0.6]} & {[0.3,0.4]} & {[0.2,0.4]} & {[0.5,0.5]}
\end{array}\right]
$$$$
\bar{R}_{2}=\left[\begin{array}{cccc}
{[0.5,0.5]} & {[0.1,0.3]} & {[0.8,0.9]} & {[0.5,0.6]} \\
{[0.7,0.9]} & {[0.5,0.5]} & {[0.7,0.9]} & {[0.9,1]} \\
{[0.1,0.2]} & {[0.1,0.3]} & {[0.5,0.5]} & {[0.8,0.9]} \\
{[0.4,0.5]} & {[0,0.1]} & {[0.1,0.2]} & {[0.5,0.5]}
\end{array}\right]
$$

287

288

$$
\bar{R}_{3}=\left[\begin{array}{cccc}
{[0.5,0.5]} & {[0.4,0.5]} & {[0.9,1]} & {[0.9,1]} \\
{[0.5,0.6]} & {[0.5,0.5]} & {[0.3,0.4]} & {[0.95,1]} \\
{[0,0.1]} & {[0.6,0.7]} & {[0.5,0.5]} & {[0.95,1]} \\
{[0,0.1]} & {[0,0.05]} & {[0,0.05]} & {[0.5,0.5]}
\end{array}\right]
$$

289 For $\bar{R}_{1}$, by using (4.1), one obtains the following transformation matrix:

290

$$
\hat{\bar{R}}_{1}=\left[\begin{array}{cccc}
{[0.5,0.5]} & {[0.37500,0.47500]} & {[0.41250,0.51250]} & {[0.51250,0.61250]} \\
{[0.52500,0.62500]} & {[0.5,0.5]} & {[0.48750,0.58750]} & {[0.58750,0.68750]} \\
{[0.48750,0.58750]} & {[0.41250,0.51250]} & {[0.5,0.5]} & {[0.50000,0.70000]} \\
{[0.38750,0.48750]} & {[0.31250,0.41250]} & {[0.30000,0.50000]} & {[0.5,0.5]}
\end{array}\right]
$$

291 Since all elements of $\hat{\bar{R}}_{1}$ are in $D([0,1])$, as per (4.2), we have $c=1$. Therefore, the 292 constructed additive consistent IFPR $\hat{\bar{R}}_{1}^{\prime}=\hat{\bar{R}}_{1}$. It can be easily verified that the widths of 293 the intervals in $\hat{\bar{R}}_{1}$ are equal to the widths of the corresponding elements in $\bar{R}_{1}$.

294 For $\bar{R}_{2}$ and $\bar{R}_{3}$, by using (4.1), the following transformation matrices are derived:

$295 \quad \hat{\bar{R}}_{2}=\left[\begin{array}{cccc}{[0.5,0.5]} & {[0.16250,0.36250]} & {[0.55000,0.65000]} & {[0.68750,0.78750]} \\ {[0.63750,0.83750]} & {[0.5,0.5]} & {[0.7375,0.9375]} & {[0.92500,1.02500]} \\ {[0.35000,0.45000]} & {[0.06250,0.26250]} & {[0.5,0.5]} & {[0.58750,0.68750]} \\ {[0.21250,0.31250]} & {[-0.0250,0.07500]} & {[0.31250,0.41250]} & {[0.5,0.5]}\end{array}\right]$ 


$$
\hat{\bar{R}}_{3}=\left[\begin{array}{cccc}
{[0.5,0.5]} & {[0.56875,0.66875]} & {[0.61875,0.71875]} & {[1.01250,1.11250]} \\
{[0.33125,0.43125]} & {[0.5,0.5]} & {[0.50000,0.60000]} & {[0.91875,0.96875]} \\
{[0.28125,0.38125]} & {[0.40000,0.50000]} & {[0.5,0.5]} & {[0.86875,0.91875]} \\
{[-0.1125,-0.0125]} & {[0.03125,0.08125]} & {[0.08125,0.13125]} & {[0.5,0.5]}
\end{array}\right]
$$

297 In $\hat{\bar{R}}_{2}$, the upper bound of $\hat{\bar{r}}_{24}$ is greater than 1 (correspondingly, the lower bound of $\hat{\bar{r}}_{42}$

298 is less than 0). In $\hat{\bar{R}}_{3}$, both the upper and lower bounds of $\hat{\bar{r}}_{14}$ are greater than 1

299 (correspondingly, the upper and lower bounds of $\hat{\bar{r}}_{41}$ are both less than 0). Based on (4.2),

300 their corresponding values of $c$ are 1.025 and 1.1125 , respectively. As such, the resulting 301 transformation functions are as follows

302

$$
\varphi\left(\left[\hat{r}_{i j}^{-}, \hat{r}_{i j}^{+}\right]\right)=\left[\frac{\hat{r}_{i j}^{-}+0.025}{1.05}, \frac{\hat{r}_{i j}^{+}+0.025}{1.05}\right] \quad \varphi\left(\left[\hat{r}_{i j}^{-}, \hat{r}_{i j}^{+}\right]\right)=\left[\frac{\hat{r}_{i j}^{-}+0.1125}{1.225}, \frac{\hat{r}_{i j}^{+}+0.1125}{1.225}\right]
$$

303

Based on (4.4), the constructed consistent IFPRs based on $\bar{R}_{2}$ and $\bar{R}_{3}$ are obtained

304 as

$$
\begin{aligned}
& \text { as } \\
& 305 \quad \hat{\bar{R}}_{2}^{\prime}=\left[\begin{array}{cccc}
{[0.5,0.5]} & {[0.17857,0.36905]} & {[0.54762,0.64286]} & {[0.67857,0.77381]} \\
{[0.63095,0.82143]} & {[0.5,0.5]} & {[0.72619,0.91667]} & {[0.90476,1.00000]} \\
{[0.35714,0.45238]} & {[0.08333,0.27381]} & {[0.5,0.5]} & {[0.58333,0.67857]} \\
{[0.22619,0.32143]} & {[0.00000,0.09524]} & {[0.32143,0.41667]} & {[0.5,0.5]}
\end{array}\right] \\
& 306 \quad \hat{\bar{R}}_{3}^{\prime}=\left[\begin{array}{cccc}
{[0.5,0.5]} & {[0.55612,0.63776]} & {[0.59694,0.67857]} & {[0.91876,1.00000]} \\
{[0.36224,0.44388]} & {[0.5,0.5]} & {[0.50000,0.58163]} & {[0.84184,0.88265]} \\
{[0.32143,0.40306]} & {[0.41837,0.50000]} & {[0.5,0.5]} & {[0.80102,0.84184]} \\
{[0.00000,0.08163]} & {[0.11735,0.15816]} & {[0.15816,0.19898]} & {[0.5,0.5]}
\end{array}\right]
\end{aligned}
$$

307 For the final constructed consistent IFPRs $\hat{\bar{R}}_{2}^{\prime}$ and $\hat{\bar{R}}_{3}^{\prime}$, computational results indicate that

308 the widths of the original interval judgments in $\bar{R}_{2}$ and $\bar{R}_{3}$ have been scaled down by a

309 factor of $1 / 1.05$ and 1/1.225, respectively. By employing (3.2), one can determine the

310 mean absolute deviations for all off-diagonal intervals between the original IFPRs and

311 their corresponding constructed consistent IFPRs as follows:

$312 d\left(\bar{R}_{1}, \hat{\bar{R}}_{1}^{\prime}\right)=0.05833, d\left(\bar{R}_{2}, \hat{\bar{R}}_{2}^{\prime}\right)=0.1246, d\left(\bar{R}_{3}, \hat{\bar{R}}_{3}^{\prime}\right)=0.15425$

\section{5. Approaches to improving consistency of IFPRs}


The proposed approach in Section 4 is able to construct an additive consistent IFPR

$315 \hat{\bar{R}}^{\prime}$ based on any given inconsistent IFPR $\bar{R}$. However, this consistency comes at a cost

316 as the mean absolute deviation between $\bar{R}$ and $\hat{\bar{R}}^{\prime}$ tends to be high. In many decision

317 situations, a DM may relax this consistency requirement as long as the inconsistency is

318 restricted to an acceptable level or the rectified IFPR possesses the weak transitivity

319 property. Presumably, this relaxation will result in an IFPR with a smaller mean absolute

320 deviation from the original IFPR $\bar{R}$. Similar to the treatment in Ma et al. (2006) for fuzzy

321 preference relations, a weighted averaging scheme combining $\hat{\bar{R}}^{\prime}$ and $\bar{R}$ is proposed as

322 follows:

$$
\tilde{R}(\lambda)=\left(\tilde{r}_{i j}(\lambda)\right)_{n \times n}=(1-\lambda) \bar{R}+\lambda \hat{\bar{R}}^{\prime}
$$

324 where $\lambda$ is a weight with $\lambda \in[0,1], \quad \hat{\bar{R}}^{\prime}=\left(\hat{\bar{r}}_{i j}^{\prime}\right)_{n \times n}$ is defined by (4.4) and $\tilde{r}_{i j}(\lambda)=(1-\lambda) \bar{r}_{i j}+\lambda \hat{\bar{r}}_{i j}^{\prime}$ for all $i, j=1,2, \ldots, n$.

326 As $\bar{R}$ and $\hat{\bar{R}}^{\prime}$ are IFPRs, according to interval arithmetic and Definition 2.1 in 327 Section 2 , it is easy to prove the following result.

Theorem 5.1 Assume that $\tilde{R}(\lambda)=\left(\tilde{r}_{i j}(\lambda)\right)_{n \times n}=\left(\left[\tilde{r}_{i j}^{-}(\lambda), \tilde{r}_{i j}^{+}(\lambda)\right]\right)_{n \times n}$ is defined by (5.1),

329 then for any $0 \leq \lambda \leq 1, \tilde{R}(\lambda)$ is an IFPR and $\tilde{r}_{i j}^{+}(\lambda)-\tilde{r}_{i j}^{-}(\lambda)=\left(1-\frac{2(c-1)}{2 c-1} \lambda\right)\left(r_{i j}^{+}-r_{i j}^{-}\right)$.

If $c=1$, it is apparent that $\tilde{r}_{i j}^{+}(\lambda)-\tilde{r}_{i j}^{-}(\lambda)=r_{i j}^{+}-r_{i j}^{-}$, i.e., the interval width for any

331 element in the original IFPR $\bar{R}$ (as well as the constructed consistent IFPR $\hat{\bar{R}}^{\prime}$ ) remains

332 the same after (5.1) is applied. If $c>1$, it is easy to verify that $\frac{1}{2 c-1} \leq 1-\frac{2(c-1)}{2 c-1} \lambda$.

333 Therefore, for any $0 \leq \lambda \leq 1, \hat{r}_{i j}^{\prime+}-\hat{r}_{i j}^{--}=\frac{r_{i j}^{+}-r_{i j}^{-}}{2 c-1} \leq\left(1-\frac{2(c-1)}{2 c-1} \lambda\right)\left(r_{i j}^{+}-r_{i j}^{-}\right)=\tilde{r}_{i j}^{+}(\lambda)-\tilde{r}_{i j}^{-}(\lambda)$

$334 \leq r_{i j}^{+}-r_{i j}^{-}$. This means that the interval width for an element in $\tilde{R}(\lambda)$ lies between that for

335 a corresponding element in the original IFPR $\bar{R}$ and that for a corresponding element in 336 the constructed consistent IFPR $\hat{\bar{R}}^{\prime}$.

337 Theorem 5.2 If $0 \leq \lambda_{1} \leq \lambda_{2} \leq 1$, then $C I\left(\tilde{R}\left(\lambda_{1}\right)\right) \leq C I\left(\tilde{R}\left(\lambda_{2}\right)\right)$. 


$$
\tilde{r}_{i j}^{-}\left(\lambda_{1}\right)+\tilde{r}_{i j}^{+}\left(\lambda_{1}\right)+\tilde{r}_{j k}^{-}\left(\lambda_{1}\right)+\tilde{r}_{j k}^{+}\left(\lambda_{1}\right)+\tilde{r}_{k i}^{-}\left(\lambda_{1}\right)+\tilde{r}_{k i}^{+}\left(\lambda_{1}\right)=
$$

$$
\left(1-\lambda_{1}\right)\left(r_{i j}^{-}+r_{i j}^{+}+r_{j k}^{-}+r_{j k}^{+}+r_{k i}^{-}+r_{k i}^{+}\right)+\lambda_{1}\left(\hat{r}_{i j}^{\prime}+\hat{r}_{i j}^{++}+\hat{r}_{j k}^{-}+\hat{r}_{j k}^{+}+\hat{r}_{k i}^{\prime-}+\hat{r}_{k i}^{+}\right) \forall i, j, k=1,2, \ldots, n
$$

$\hat{r}_{j k}^{\prime+}+\hat{r}_{k i}^{\prime-}+\hat{r}_{k i}^{++}=3 \forall i, j, k=1,2, \ldots, n$. Then

$$
\begin{aligned}
& C I\left(\tilde{R}\left(\lambda_{1}\right)\right)=1-\frac{1}{3 n(n-1)(n-2)} \sum_{i=1}^{n} \sum_{j=1, j \neq i}^{n} \sum_{k=1, k \neq i, j}^{n}\left(\mid \tilde{r}_{i j}^{-}\left(\lambda_{1}\right)+\tilde{r}_{i j}^{+}\left(\lambda_{1}\right)+\tilde{r}_{j k}^{-}\left(\lambda_{1}\right)+\tilde{r}_{j k}^{+}\left(\lambda_{1}\right)+\tilde{r}_{k i}^{-}\left(\lambda_{1}\right)+\right. \\
& \left.\tilde{r}_{k i}^{+}\left(\lambda_{1}\right)-3 \mid\right)=1-\frac{1-\lambda_{1}}{3 n(n-1)(n-2)} \sum_{i=1}^{n} \sum_{j=1, j \neq i}^{n} \sum_{k=1, k \neq i, j}^{n}\left(\left|r_{i j}^{-}+r_{i j}^{+}+r_{j k}^{-}+r_{j k}^{+}+r_{k i}^{-}+r_{k i}^{+}-3\right|\right)
\end{aligned}
$$

Similarly,

$$
C I\left(\tilde{R}\left(\lambda_{2}\right)\right)=1-\frac{1-\lambda_{2}}{3 n(n-1)(n-2)} \sum_{i=1}^{n} \sum_{j=1, j \neq i}^{n} \sum_{k=1, k \neq i, j}^{n}\left(\left|r_{i j}^{-}+r_{i j}^{+}+r_{j k}^{-}+r_{j k}^{+}+r_{k i}^{-}+r_{k i}^{+}-3\right|\right)
$$

As $0 \leq \lambda_{1} \leq \lambda_{2} \leq 1$, one can obtain that $C I\left(\tilde{R}\left(\lambda_{1}\right)\right) \leq C I\left(\tilde{R}\left(\lambda_{2}\right)\right)$.

Theorem 5.2 indicates that $C I(\tilde{R}(\lambda))$ is an increasing function in $\lambda \in[0,1]$.

Theorem 5.3 Let $\bar{R}=\left(\bar{r}_{i j}\right)_{n \times n}$ be an IFPR with an unacceptable consistency level, and

$348 t$ be an acceptable consistency threshold. If $\frac{t-C I(\bar{R})}{1-C I(\bar{R})} \leq \lambda \leq 1$, then $\tilde{R}(\lambda)$ is an IFPR with acceptable consistency.

Proof. Since

and

$$
C I(\bar{R})=1-\frac{1}{3 n(n-1)(n-2)} \sum_{i=1}^{n} \sum_{j=1, j \neq i}^{n} \sum_{k=1, k \neq i, j}^{n}\left(\left|r_{i j}^{-}+r_{i j}^{+}+r_{j k}^{-}+r_{j k}^{+}+r_{k i}^{-}+r_{k i}^{+}-3\right|\right),
$$

$$
C I(\tilde{R}(\lambda))=1-\frac{1-\lambda}{3 n(n-1)(n-2)} \sum_{i=1}^{n} \sum_{j=1, j \neq i}^{n} \sum_{k=1, k \neq i, j}^{n}\left(\left|r_{i j}^{-}+r_{i j}^{+}+r_{j k}^{-}+r_{j k}^{+}+r_{k i}^{-}+r_{k i}^{+}-3\right|\right)
$$

354 we have $C I(\tilde{R}(\lambda))=C I(\bar{R})+\lambda(1-C I(\bar{R}))$. Therefore, if $\frac{t-C I(\bar{R})}{1-C I(\bar{R})} \leq \lambda \leq 1$, one can 355 ascertain that $C I(\tilde{R}(\lambda)) \geq t$.

356 By (5.1) and Theorem 5.2, one can see that if $\lambda \rightarrow 0, \tilde{R}(\lambda) \rightarrow \bar{R}$, indicating that the 357 closer the repaired IFPR $\tilde{R}(\lambda)$ reflects the original preference relation $\bar{R}$. However, the 
358 consistency level of $\tilde{R}(\lambda)$ will be lower. On the other hand, if $\lambda \rightarrow 1, \tilde{R}(\lambda) \rightarrow \hat{\bar{R}}$, 359 implying that the closer $\tilde{R}(\lambda)$ is to the constructed additive consistent IFPR $\hat{\bar{R}}^{\prime}$.

360 Similarly, in this case, $\tilde{R}(\lambda)$ deviates further from the original IFPR $\bar{R}$. Therefore,

361 according to Theorem 5.3, for a given IFPR $\bar{R}$ and an acceptable consistency threshold $t$, 362 a sensible way to repair $\bar{R}$ is to apply (5.1) by setting

$$
\lambda=\frac{t-C I(\bar{R})}{1-C I(\bar{R})}
$$

364 In this case, it is guaranteed that the modified IFPR $\tilde{R}(\lambda)$ has an acceptable consistency

365 level and reflects the DM's original preference relation in $\bar{R}$ as much as possible.

366 Example 2. For the three original IFPRs $\bar{R}_{1}, \bar{R}_{2}, \bar{R}_{3}$ in Example 1, assume that an 367 acceptable consistency threshold is established as $t=0.85$. By Definition 3.1, one has $368 C I\left(\bar{R}_{1}\right)=0.9>t, C I\left(\bar{R}_{2}\right)=0.78333<t$ and $C I\left(\bar{R}_{3}\right)=0.78333<t . \quad$ Example 1 indicates 369 that $\bar{R}_{1}$ is additive inconsistent. However, if the DM can accept certain inconsistency as 370 reflected in the threshold $t=0.85$, the consistency level of $\bar{R}_{1}$ is deemed acceptable, but

$371 \bar{R}_{2}$ and $\bar{R}_{3}$ are deemed to have unacceptable consistency. In this case, their consistency

372 levels have to be improved to reach the acceptable threshold by using (5.1) where $\hat{\bar{R}}_{2}^{\prime}$ and

$373 \hat{\bar{R}}_{3}^{\prime}$ are the corresponding consistent IFPR obtained in Example 1 and $\lambda$ is determined by 374 (5.2).

375 Given that the $\bar{R}_{2}$ and $\bar{R}_{3}$ have the same consistency index, by using (5.2), we have $376 \lambda=0.3077$ for both IFPRs. As per $(5.1)$, one can obtain $\tilde{R}_{2}(0.3077)=0.6923 \bar{R}_{2}+$ $3770.3077 \hat{\bar{R}}_{2}^{\prime}$ and $\tilde{R}_{3}(0.3077)=0.6923 \bar{R}_{3}+0.3077 \hat{\bar{R}}_{3}^{\prime}$ as follows

$378 \quad \tilde{R}_{2}(0.3077)=\left[\begin{array}{cccc}{[0.5,0.5]} & {[0.12418,0.32125]} & {[0.72234,0.82088]} & {[0.55495,0.65348]} \\ {[0.67875,0.87582]} & {[0.5,0.5]} & {[0.70806,0.90513]} & {[0.90146,1.00000]} \\ {[0.17912,0.27766]} & {[0.09487,0.29194]} & {[0.5,0.5]} & {[0.73333,0.83187]} \\ {[0.34652,0.44505]} & {[0.00000,0.09854]} & {[0.16813,0.26667]} & {[0.5,0.5]}\end{array}\right]$ 379 


$$
\tilde{R}_{3}(0.3077)=\left[\begin{array}{cccc}
{[0.5,0.5]} & {[0.44804,0.54239]} & {[0.80675,0.90110]} & {[0.90565,1.00000]} \\
{[0.45761,0.55196]} & {[0.5,0.5]} & {[0.36154,0.45589]} & {[0.91672,0.96389]} \\
{[0.09890,0.19325]} & {[0.54411,0.63846]} & {[0.5,0.5]} & {[0.90416,0.95133]} \\
{[0.00000,0.09435]} & {[0.03611,0.08328]} & {[0.04867,0.09584]} & {[0.5,0.5]}
\end{array}\right]
$$

One can verify that $C I\left(\tilde{R}_{2}(0.3077)\right)=C I\left(\tilde{R}_{3}(0.3077)\right)=0.85 \geq t$. Therefore, after

382 applying $(5.1)$, the resulting $\tilde{R}_{2}(0.3077)$ and $\tilde{R}_{3}(0.3077)$ are two rectified IFPRs with 383 acceptable consistency.

384 It should be noted that, if the DM is willing to accept limited inconsistency in a 385 rectified IFPR $\tilde{R}$, its mean absolute deviation from the original IFPR $\bar{R}$ should be 386 smaller than that between a constructed consistent IFPR $\hat{\bar{R}}^{\prime}$ and $\bar{R}$. For instance, by 387 using (3.2), one can verify that $d\left(\bar{R}_{2}, \tilde{R}_{2}(0.3077)\right)=0.03834<d\left(\bar{R}_{2}, \hat{\bar{R}}_{2}^{\prime}\right)=0.1246$ and $388 d\left(\bar{R}_{3}, \tilde{R}_{3}(0.3077)\right)=0.04746<d\left(\bar{R}_{3}, \hat{\bar{R}}_{3}^{\prime}\right)=0.15425$. Furthermore, computational results 389 confirm a reduction ratio of 0.98535 between the interval width of an element in $\bar{R}_{2}$ and 390 that of the corresponding element in $\tilde{R}_{2}(0.3077)$. Similarly, the reduction ratio is 0.94348

391 between the interval width of each element in $\bar{R}_{3}$ and that of the corresponding element

392 in $\tilde{R}_{3}(0.3077)$. On the other hand, the corresponding ratios are $\frac{1}{1.05}=0.95238$ and $393 \frac{1}{1.225}=0.81633$ for the additive consistent IFPRs $\hat{\bar{R}}_{2}^{\prime}$ and $\hat{\bar{R}}_{3}^{\prime}$, respectively. This result 394 indicates that, if the consistency requirement can be relaxed to an acceptable consistency 395 threshold, one can obtain a modified IFPR that is closer to the original IFPR in terms of 396 both the mean absolute deviation and the interval uncertainty as reflected in the interval 397 width.

398 According to Definition 2.3 , one can verify that IFPRs $\bar{R}_{1}, \bar{R}_{3}$ and $\tilde{R}_{3}(0.3077)$ are not 399 weakly transitive, but $\bar{R}_{2}$ and $\tilde{R}_{2}(0.3077)$ are. This indicates that there does not exist 400 definite inclusion relationship between weak transitivity and acceptable consistency. For 401 instance, $\bar{R}_{2}$ is weakly transitive, but at $t=0.85$, its consistency level is unacceptable. On 402 the other hand, as long as $t<1$, an IFPR with acceptable consistency is not necessarily 
403 weakly transitive. For example, $\tilde{R}_{3}(0.3077)$ is an IFPR with acceptable consistency at $t=$ 4040.85 , but it is not weakly transitive due to $p\left(\tilde{r}_{21} \geq[0.5,0.5]\right)=0.5507>0.5$, $405 p\left(\tilde{r}_{13} \geq[0.5,0.5]\right)=1>0.5$, but $p\left(\tilde{r}_{23} \geq[0.5,0.5]\right)=0<0.5$. Given that the consistency 406 index increases in $\lambda$ (Theorem 5.2) and, if $t=1$ or $\lambda=1, \tilde{R}=\hat{\bar{R}}^{\prime}$ is additive consistent 407 (Theorem 4.3) and, hence, weakly transitive (Theorem 3.1), it is possible to obtain a 408 rectified IFPR with both acceptable consistency and weak transitivity by increasing the 409 value of $\lambda$. Next, we shall turn our attention to put forward a framework to repair an 410 inconsistent IFPR, thereby obtaining a rectified IFPR with both acceptable consistency 411 and weak transitivity.

412 In the following, assume that a DM provides his/her IFPR with strict comparison 413 information, i.e., $p\left(\bar{r}_{i j} \geq[0.5,0.5]\right) \neq 0.5$ for all $i, j=1,2, \ldots, n$ and $i \neq j$. Then, for every 414 IFPR, its associated preference matrix can be defined as follows.

415 Definition 5.1 The preference matrix $Q=\left(q_{i j}\right)_{n \times n}$ of an IFPR $\bar{R}=\left(\bar{r}_{i j}\right)_{n \times n}$ is defined as

$$
q_{i j}=\left\{\begin{array}{ll}
1, & p\left(\bar{r}_{i j} \geq[0.5,0.5]\right)>0.5 \\
0, & \text { otherwise }
\end{array} \quad i, j=1,2, \ldots, n\right.
$$

The preference matrix $Q$ expresses the DM's strict preference relations on $X$ 418 without considering preference degrees. $q_{i j}=1$ indicates that the DM prefers $x_{i}$ to $x_{j}$, 419 while $q_{i j}=0$ means that the DM prefers $x_{j}$ to $x_{i}$. As $p\left(\bar{r}_{i j} \geq[0.5,0.5]\right) \neq 0.5$, the 420 elements in $Q$ satisfy $q_{i j}+q_{j i}=1$ for all $i, j=1,2, \ldots, n, i \neq j$.

$421 \quad$ Let

$$
q_{i}=\sum_{j=1}^{n} q_{i j}, \quad i=1,2, \ldots, n
$$

423 It is obvious that $0 \leq q_{i} \leq n-1$ for any $i=1,2, \ldots, n$, and $\sum_{i=1}^{n} q_{i}=\frac{n(n-1)}{2}$.

424 Preference matrices here can model team tournaments with $q_{i j}$ characterizing 425 whether team $x_{i}$ defeats $x_{j}$, where $q_{i j}=1$ indicates that $x_{i}$ defeats $x_{j}$ and $q_{i j}=0$ 426 describes that $x_{j}$ defeats $x_{i}$, and no ties are allowed. According to the likelihood 
427 property (e) in Section 2, $p\left(\bar{r}_{i j} \geq[0.5,0.5]\right)>0.5$ implies $\frac{r_{i j}^{-}+r_{i j}^{+}}{2}>0.5$. Therefore, the 428 preference matrix $Q$ here is equivalent to a preference matrix under the fuzzy preference 429 relation $P=\left(p_{i j}\right)_{n \times n}$ in Ma et al. (2006), where $p_{i j}=\frac{r_{i j}^{-}+r_{i j}^{+}}{2}$. According to Corollary 3.3 430 and Proposition 1 in Ma et al. (2006), the following judgment methods can be established 431 for weak transitivity.

432 Corollary 5.1 Let $\bar{R}=\left(\bar{r}_{i j}\right)_{n \times n}$ be an IFPR and $Q=\left(q_{i j}\right)_{n \times n}$ be its associated 433 preference matrix with $q_{i j}+q_{j i}=1$ for all $i, j=1,2, \ldots, n, i \neq j$. Then $\bar{R}$ is weakly 434 transitive if and only if $S=0$, where $S$ is defined as (Ma et al. 2006)

$$
S=\frac{n(n-1)(n-2)}{6}-\frac{1}{2} \sum_{i=1}^{n} q_{i}\left(q_{i}-1\right) .
$$

436 Alternatively, another method is furnished below to tell whether an IFPR is weakly transitive based on its associated preference matrix.

438 Corollary 5.2 Let $\bar{R}=\left(\bar{r}_{i j}\right)_{n \times n}$ be an IFPR and $Q=\left(q_{i j}\right)_{n \times n}$ be its associated 439 preference matrix. Then $\bar{R}$ is weakly transitive if and only if $n$ values $q_{i}(i=1,2, \ldots, n)$ 440 can be ordered as $\{n-1, n-2, \ldots, 1,0\}$.

441 Proof. First, we prove sufficiency. As $q_{i}(i=1,2, \ldots, n)$ can be ordered as $442\{n-1, n-2, \ldots, 1,0\}$, we have $\sum_{i=1}^{n}\left(q_{i}\right)^{2}=\sum_{l=0}^{n-1} l^{2}=\frac{n(n-1)(2 n-1)}{6}$ and $\sum_{i=1}^{n} q_{i}=\frac{n(n-1)}{2}$. 443 Then, $S=\frac{n(n-1)(n-2)}{6}-\frac{1}{2} \sum_{i=1}^{n} q_{i}\left(q_{i}-1\right)=0$. By Corollary 5.1, $\bar{R}$ is weakly transitive.

444 Next, we prove the necessary part. Since $\bar{R}$ is weakly transitive, by Corollary 5.1, it 445 follows that $\sum_{i=1}^{n}\left(q_{i}\right)^{2}=\frac{n(n-1)(n-2)}{3}+\sum_{i=1}^{n} q_{i}$. On the other hand, as per Definition 5.1 and 446 Eq. (5.4), one has $0 \leq q_{i} \leq n-1$ for any $i=1,2, \ldots, n$, and $\sum_{i=1}^{n} q_{i}=\frac{n(n-1)}{2}$. Thus, 
$447 \sum_{i=1}^{n}\left(q_{i}\right)^{2}=\frac{n(n-1)(2 n-1)}{6}$. Therefore, the $n$ values $q_{i}(i=1,2, \ldots, n)$ can be arranged as $448 \quad\{n-1, n-2, \ldots, 1,0\}$.

449 If $\bar{R}$ is weakly transitive, then the rank order of the objects on $X$ is the same as the 450 ordering of $q_{i}(i=1,2, \ldots, n)$.

451 Based on Theorem 5.3, if an IFPR $\bar{R}$ given by a DM is inconsistent, it can be 452 converted to $\tilde{R}(\lambda)$ with acceptable consistency by using $(5.1)$, where $\lambda=\frac{t-C I(\bar{R})}{1-C I(\bar{R})}$. If $453 \tilde{R}(\lambda)$ is not weakly transitive, one can increase the value of $\lambda$ to obtain a rectified IFPR 454 with both acceptable consistency and weak transitivity.

455 Based on the aforesaid analyses, the following algorithm is formulated to improve 456 the consistency of an IFPR.

457 Algorithm: Let $\bar{R}=\left(\bar{r}_{i j}\right)_{n \times n}$ be an original IFPR and $t$ be an acceptable consistency 458 threshold given by a DM. The iteration procedure is described as follows.

459 Step 1. Establish the transformation matrix $\hat{\bar{R}}=\left(\left[\hat{r}_{i j}^{-}, \hat{r}_{i j}^{+}\right]\right)_{n \times n}$ as per (4.1).

460 Step 2. Compute the value $c$ by using (4.2).

461 Step 3. Construct the additive consistent IFPR $\hat{\bar{R}}^{\prime}=\left(\hat{\bar{r}}^{\prime}\right)_{n \times n}$ as per (4.4).

Step 4. Set $k=0$ and calculate $\lambda_{k}=\max \left(\frac{t-C I(\bar{R})}{1-C I(\bar{R})}, 0\right)$, where $C I(\bar{R})$ is determined 463 by (3.1).

Step 5. Derive the weighted IFPR $\tilde{R}^{(k)}$ by $\tilde{R}^{(k)}=\left(1-\lambda_{k}\right) \bar{R}+\lambda_{k} \hat{\bar{R}}^{\prime}$;

470 that $0<\lambda_{k}+\delta \leq 1$. To make the derived IFPR as close to the original IFPR as possible, a 471 reasonably small value for $\delta$ is needed. Without loss of generality, set $0.01 \leq \delta \leq 0.1$. 
Step 10. If $\lambda_{k}<1$, go to step 5; otherwise, let $\tilde{R}^{(k)}=\hat{\bar{R}}^{\prime}$, and go to step 6;

Step 11. Output $k, \tilde{R}^{(k)}, Q^{(k)},\left(q_{1}^{(k)}, q_{2}^{(k)}, \ldots, q_{n}^{(k)}\right), S^{(k)}$;

Next, it is ascertained that this algorithm will terminate after a finite number of iterations.

477 Theorem 5.4 Assume that $\bar{R}=\left(\bar{r}_{i j}\right)_{n \times n}$ is an inconsistent IFPR, then a rectified IFPR 478 with acceptable consistency and weak transitivity will be obtained after applying the above algorithm to $\bar{R}$ for a finite number of iterations.

$480 \quad$ Proof. For a given consistency threshold $t$ and step size $\delta$, there exists a natural 481 number $N$ such that $\lambda_{0}+N \cdot \delta \geq 1$. Then after $N$ iterations, we have $\tilde{R}^{(N)}=\hat{\bar{R}}^{\prime}$. It follows 482 from Corollary 4.1 that $\tilde{R}^{(N)}$ is an additive consistent IFPR and, hence, weakly 483 transitive as per Theorem 3.1. On the other hand, if the algorithm terminates after $k$ 484 iterations where $k<N$, then $\tilde{R}^{(k)}$ is an IFPR with weak transitivity. Moreover, as 485 $\lambda_{k}=\lambda_{0}+k \cdot \delta \geq \lambda_{0}$, it follows from Theorem 5.3 that $\tilde{R}^{(k)}$ has acceptable consistency. Therefore, it is ascertained that a rectified IFPR with acceptable consistency and weak transitivity can always be obtained after a finite number of iterations.

488 Example 3. Assume that a DM conducts an exhaustive pairwise comparison on an 489 alternative set $X=\left\{x_{1}, x_{2}, x_{3}, x_{4}\right\}$, and the result is given as the following IFPR:

$$
\bar{R}=\left[\begin{array}{cccc}
{[0.5,0.5]} & {[0.8,1]} & {[0.7,0.9]} & {[0.5,0.9]} \\
{[0,0.2]} & {[0.5,0.5]} & {[0.5,0.7]} & {[0.7,0.9]} \\
{[0.1,0.3]} & {[0.3,0.5]} & {[0.5,0.5]} & {[0.6,0.8]} \\
{[0.1,0.5]} & {[0.1,0.3]} & {[0.2,0.4]} & {[0.5,0.5]}
\end{array}\right]
$$

Without loss of generality, let $t=0.85$. In the following, the proposed algorithm is 492 applied to improve the consistency level of $\bar{R}$.

$493 \quad$ As per (4.1), the transformation matrix $\hat{\bar{R}}=\left(\left[\hat{r}_{i j}^{-}, \hat{r}_{i j}^{+}\right]\right)_{4 \times 4}$ is established as

$$
\hat{\bar{R}}=\left[\begin{array}{cccc}
{[0.5,0.5]} & {[0.6,0.7]} & {[0.55,0.65]} & {[0.65,1.05]} \\
{[0.3,0.4]} & {[0.5,0.5]} & {[0.35,0.55]} & {[0.65,0.75]} \\
{[0.35,0.45]} & {[0.45,0.65]} & {[0.5,0.5]} & {[0.7,0.8]} \\
{[-0.05,0.35]} & {[0.25,0.35]} & {[0.2,0.3]} & {[0.5,0.5]}
\end{array}\right]
$$


496 the following IFPR with additive consistency is constructed as per (4.4).

497

$$
\hat{\bar{R}}^{\prime}=\left[\begin{array}{cccc}
{[0.5,0.5]} & {[0.59091,0.68182]} & {[0.54545,0.63636]} & {[0.63636,1.00000]} \\
{[0.31818,0.40909]} & {[0.5,0.5]} & {[0.36364,0.54545]} & {[0.63636,0.72727]} \\
{[0.36364,0.45455]} & {[0.45455,0.63636]} & {[0.5,0.5]} & {[0.68182,0.77273]} \\
{[0.00000,0.36364]} & {[0.27273,0.36364]} & {[0.22727,0.31818]} & {[0.5,0.5]}
\end{array}\right]
$$

$499 \lambda_{0}=\max \left(\frac{t-C I(\bar{R})}{1-C I(\bar{R})}, 0\right)=0.25$. Therefore, an acceptably consistent IFPR $\tilde{R}(0.25)$ is 500 constructed by (5.2) with $\lambda=\lambda_{0}$.

501

$$
\tilde{R}(0.25)=\left[\begin{array}{cccc}
{[0.5,0.5]} & {[0.82273,0.92046]} & {[0.43636,0.53409]} & {[0.53409,0.92500]} \\
{[0.07955,0.17727]} & {[0.5,0.5]} & {[0.46591,0.66136]} & {[0.75909,0.85682]} \\
{[0.46591,0.56364]} & {[0.33864,0.53409]} & {[0.5,0.5]} & {[0.69546,0.79318]} \\
{[0.07500,0.46591]} & {[0.14318,0.24091]} & {[0.20682,0.30455]} & {[0.5,0.5]}
\end{array}\right]
$$

503 By (5.3), the preference matrix $Q^{(0)}=\left(q_{i j}\right)_{4 \times 4}$ of the IFPR $\tilde{R}^{(0)}$ is derived as follows.

504

$$
Q^{(0)}=\left[\begin{array}{llll}
0 & 1 & 0 & 1 \\
0 & 0 & 1 & 1 \\
1 & 0 & 0 & 1 \\
0 & 0 & 0 & 0
\end{array}\right]
$$

505 Either Corollary 5.1 or 5.2 can be applied to judge if $\tilde{R}^{(0)}$ is weakly transitive. As 506 per (5.4), we have $q_{1}^{(0)}=2, q_{2}^{(0)}=2, q_{3}^{(0)}=2, q_{4}^{(0)}=0$. By $(5.5)$, one obtains $S^{(0)}=1 \neq 0$.

$507 \quad$ From Corollary 5.1 or 5.2 , one can see that $\tilde{R}^{(0)}$ is not weakly transitive. In fact, for 508 preference matrix $Q^{(0)}=\left(q_{i j}\right)_{4 \times 4}$, one can get a cyclic preference relation, $x_{1} \succ x_{2} \succ x_{3} \succ x_{1}$.

509 Thus the weight $\lambda$ in the rectifying formula (5.1) should be increased. Let $\delta=0.02$, one 510 can obtain a rectified IFPR $\tilde{R}^{(6)}$ with both weak transitivity and acceptable consistency.

511 The iterative process to improving consistency for the IFPR $\bar{R}$ is described in Table 1.

512 It can be seen from Table 1 that $\operatorname{CI}\left(\tilde{R}^{(0)}\right)<\operatorname{CI}\left(\tilde{R}^{(1)}\right)<\cdots<C I\left(\tilde{R}^{(6)}\right)$ and $513 d\left(\bar{R}, \tilde{R}^{(0)}\right)>d\left(\bar{R}, \tilde{R}^{(1)}\right)>\cdots>d\left(\bar{R}, \tilde{R}^{(6)}\right)$. This is understandable: as the iteration process 
514 continues, the consistency level of the resulting IFPR $\tilde{R}^{(k)}$ increases, but this comes at a 515 cost with a greater deviation from the original IFPR $\bar{R}$. One can also see from the third 516 last column that the rectified IFPR $\tilde{R}^{(k)}$ does not achieve weak transitivity until $k=6$.

517 Table 1. The process to improving consistency of IFPR $\bar{R}$

518 Iteration $k \quad$ Iterative preference relation $\tilde{R}^{(k)} \quad S^{(k)} \quad C I\left(\tilde{R}^{(k)}\right) d\left(\bar{R}, \tilde{R}^{(k)}\right)$

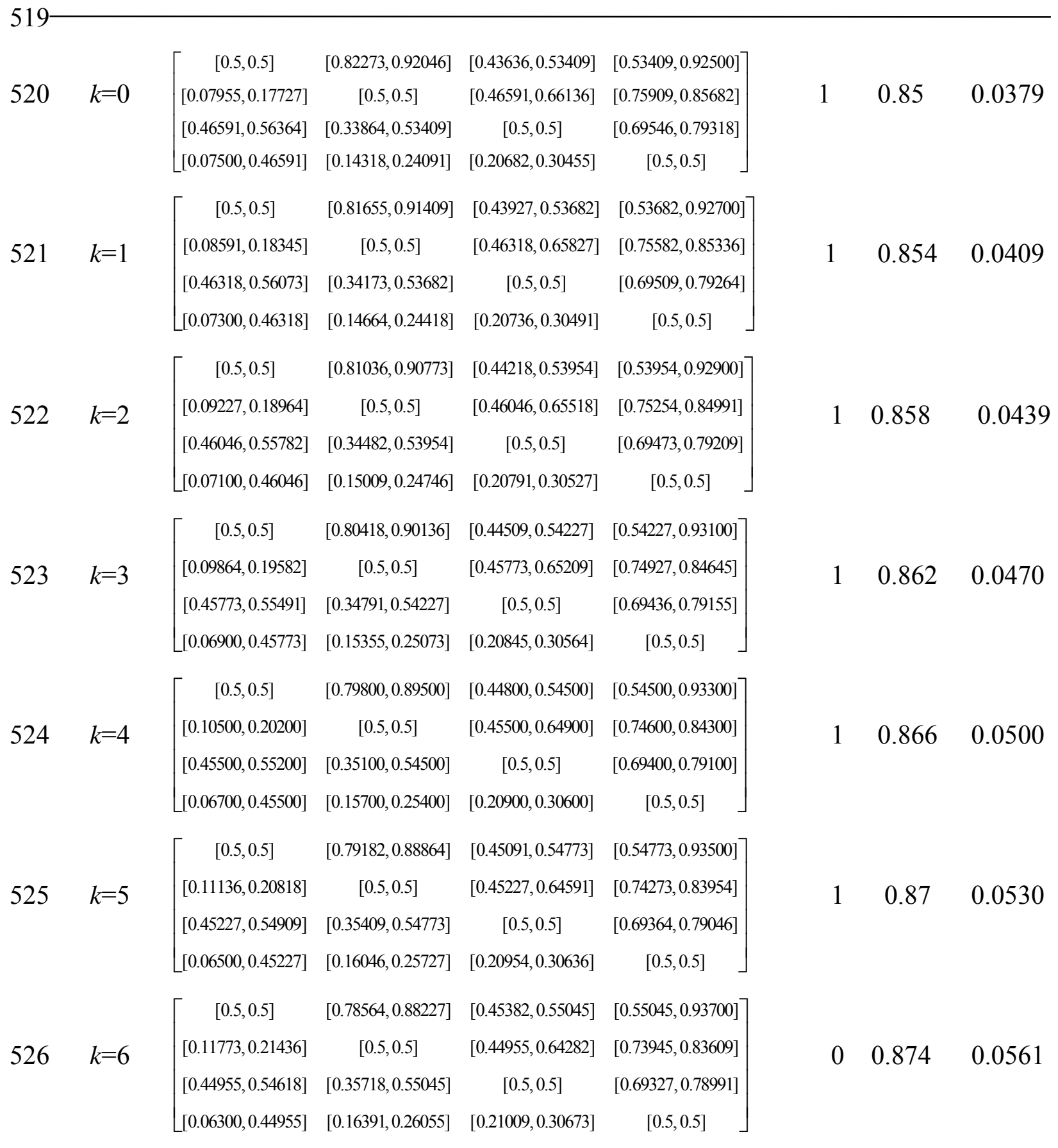


528 Next, the approach proposed by Xu (2011) is employed to improve consistency for the same IFPR $\bar{R}$ as a comparison.

530 Based on the additive consistency definition (Xu and Chen 2008), Xu (2011) develops

531 a linear program (see (M-2) on page 3901) to construct a consistent IFPR from an

532 inconsistent IFPR. Plugging $\bar{R}$ into (M-2) in Xu (2011) and solving this model, one can

533 get the optimal nonzero deviation values $\dot{d}_{24}^{-}=0.1333, \dot{d}_{34}^{-}=0.0333, \dot{d}_{42}^{+}=0.1333$ and

$534 \dot{d}_{43}^{+}=0.0333$. By (21) in Xu (2011), we obtain the following constructed IFPR with Xu 535 and Chen (2008)'s additive consistency.

$$
\hat{\bar{R}}^{X u}=\left[\begin{array}{cccc}
{[0.5,0.5]} & {[0.8,1]} & {[0.7,0.9]} & {[0.5,0.9]} \\
{[0,0.2]} & {[0.5,0.5]} & {[0.5,0.7]} & {[0.5667,0.9]} \\
{[0.1,0.3]} & {[0.3,0.5]} & {[0.5,0.5]} & {[0.5667,0.8]} \\
{[0.1,0.5]} & {[0.1,0.4333]} & {[0.2,0.4333]} & {[0.5,0.5]}
\end{array}\right]
$$

537 By Definition 2.3, one can easily verify that $\hat{\bar{R}}^{x u}$ has weak transitivity. On the other 538 hand, as per (3.1) and (3.2), one has $C I\left(\hat{\bar{R}}^{\Upsilon u}\right)=0.8389$ and $d\left(\bar{R}, \hat{\bar{R}}^{\chi u}\right)=0.0139$.

539 Although $\hat{\bar{R}}^{\text {Хu }}$ is weakly transitive and $d\left(\bar{R}, \hat{\bar{R}}^{X u}\right)<d\left(\bar{R}, \tilde{R}^{(k)}\right)$ for all $k=0,1, \ldots, 6, \hat{\bar{R}}^{\text {хu }}$

540 does not possess acceptable consistency under (3.1) as $C I\left(\hat{\bar{R}}^{X u}\right)<t=0.85$. This

541 difference is resulted from the fact that the two rectification approaches employ different

542 additive consistency constraints. The constraint in $\mathrm{Xu}$ (2011) is established by the

543 feasible region model and the proposed method herein is based on interval arithmetic.

\section{6. Conclusion}

545 Based on the additive consistency definition proposed by Wang and Li (2012), this 546 article begins with presenting new properties for additive consistent IFPRs. Then, a 547 consistency index is defined to measure the level of consistency for IFPRs, which can be 548 conveniently applied to check whether an IFPR is consistent. Subsequently, an innovative 549 approach is developed to construct an additive consistent IFPR from any inconsistent 550 IFPR. By introducing a weighted averaging scheme that integrates the original and the 551 constructed consistent IFPRs, a novel approach is put forward to improve consistency of 552 IFPRs. An iterative algorithm is then established to repair an inconsistent IFPR to derive 553 a rectified IFPR with both acceptable consistency and weak transitivity. 
554 The basic modeling principle is to ensure that the derived IFPRs can improve 555 consistency and, simultaneously, retain as much the initial interval uncertainty (measured

556 by interval widths) as possible. Numerical examples are presented to demonstrate how to 557 apply the proposed approaches. Further research is required to accommodate the cases 558 when IFPRs contain missing judgment data and induced preference matrix $Q$ includes 559 indifference relations.

\section{REFERENCES}

561 [1] Chen Y. H., Chao R. J. (2012). Supplier selection using consistent fuzzy preference relations, Expert Systems with Applications, 39: 3233-3240.

563 [2] Chiclana F., Herrera-Viedma E., Herrera F., Alonso S. (2007). Some induced ordered problems based on fuzzy preference relations. European Journal Operational Research, 182: 383-399.

[3] Dong Q., Saaty T. L. (2014). An analytic hierarchy process model of group consensus. Journal of Systems Science and Systems Engineering, 23: 362-374.

[4] Durbach I. N., Stewart T. J. (2012). Modeling uncertainty in multi-criteria decision analysis. European Journal of Operational Research, 223: 1-14.

[5] Genç S., Boran F. E., Akay D., Xu Z. (2010). Interval multiplicative transitivity for consistency, missing values and priority weights of interval fuzzy preference relations. Information Sciences, 180: 4877-4891.

[6] Herrera-Viedma E., Herrera F., Chiclana F., Luque M. (2004). Some issues on consistency of fuzzy preference relations. European Journal Operational Research, 154: $98-109$.

[7] Herrera-Viedma E., Chiclana F., Herrera F., Alonso S. (2007). Group decisionmaking model with incomplete fuzzy preference relations based on additive consistency. IEEE Transactions on Systems, Man, and Cybernetics - Part B: Cybernetics, 37: 176-189.

[8] Hu M., Ren P., Lan J., Wang J., Zheng W. (2014). Note on "Some models for deriving the priority weights from interval fuzzy preference relations". European Journal of Operational Research, 237: 771-773. 
584 [9] Lan J., Hua M., Ye X., Sun S. (2012). Deriving interval weights from an interval 585 multiplicative consistent fuzzy preference relation. Knowledge-Based Systems, 26: $586 \quad 128-134$.

587 [10] Li X., Chen X. (2014). Extension of the TOPSIS method based on prospect theory 588 and trapezoidal intuitionistic fuzzy numbers for group decision making. Journal of Systems Science and Systems Engineering, 23: 231-247.

590 [11] Liu F., Zhang W. G., Fu J. H. (2012). A new method of obtaining the priority weights from an interval fuzzy preference relation. Information Sciences 185: 32-42.

[12] Liu X., Pan Y., Xu Y., Yu S. (2012). Least square completion and inconsistency repair methods for additively consistent fuzzy preference relations. Fuzzy Sets and Systems, 198: 1-19.

[13] Ma J., Fan Z. P., Jiang Y. P., Mao J.Y., Ma L. (2006). A method for repairing the inconsistency of fuzzy preference relations. Fuzzy Sets and Systems, 157: 20-33.

[14] Saaty T. L. (1980). The Analytic Hierarchy Process. McGraw-Hill, New York.

[15] Wang Z. J. (2014). A note on "Incomplete interval fuzzy preference relations and their applications"'. Computers \& Industrial Engineering, 77: 65-69.

[16] Wang Z. J., Chen Y. G. (2014). Logarithmic least squares prioritization and completion methods for interval fuzzy preference relations based on geometric transitivity. Information Sciences, 289: 59-75.

[17] Wang Z. J., Li K. W. (2012). Goal programming approaches to deriving interval weights based on interval fuzzy preference relations. Information Sciences, 193: 180198.

[18] Wang Z. J., Li K. W. (2014). A multi-step goal programming approach for group decision making with incomplete interval additive reciprocal comparison matrices. European Journal of Operational Research, Doi: 10.1016/j.ejor.2014.10.025.

[19] Xia M., Xu Z., Chen J. (2013). Algorithms for improving consistency or consensus of reciprocal [0, 1]-valued preference relations. Fuzzy Sets and Systems, 216: 108-

612 [20] Xia M., Xu Z. (2014). Interval weight generation approaches for reciprocal relations. 613 Applied Mathematical Modelling, 38: 828-838. 
614 [21] Xu Y., Li K. W., Wang H. (2014). Consistency test and weight generation for 615 additive interval fuzzy preference relations. Soft Computing, 18: 1499-1513.

616 [22] Xu Z. (2004). On compatibility of interval fuzzy preference relations. Fuzzy 617 Optimization and Decision Making, 3: 217-225.

618 [23] Xu Z. (2007). A survey of preference relations. International Journal of General 619 Systems, 36: 179-203.

620 [25] Xu Z. (2011). Consistency of interval fuzzy preference relations in group decision 621 making. Applied Soft Computing, 11: 3898-3909.

622 [26] Xu Z., Chen J. (2008). Some models for deriving the priority weights from interval 623 fuzzy preference relations. European Journal of Operational Research, 184: 266-280. 624 [27] Yu S., Xu Z. (2014). Aggregation and decision making using intuitionistic 625 multiplicative triangular fuzzy information. Journal of Systems Science and Systems 626 Engineering, 23: 20-38 